\title{
A simple enzymatic assay for the quantification of C1-specific cellulose oxidation by lytic polysaccharide monooxygenases
}

\author{
M. B. Keller • C. Felby $\cdot$ C. A. Labate $\cdot$ V. O. A. Pellegrini $\cdot$ P. Higasi $\cdot$ \\ R. K. Singh · I. Polikarpov $\cdot$ B. M. Blossom $\mathbb{B}$
}

Received: 9 August 2019/Accepted: 8 November 2019/Published online: 20 November 2019

(C) The Author(s) 2019

\begin{abstract}
Objective The development of an enzymatic assay for the specific quantification of the C1-oxidation product, i.e. gluconic acid of cellulose active lytic polysaccharide monooxygenases (LPMOs).

Results In combination with a $\beta$-glucosidase, the spectrophotometrical assay can reliably quantify the specific C1- oxidation product of LPMOs acting on cellulose. It is applicable for a pure cellulose model substrate as well as lignocellulosic biomass. The enzymatic assay compares well with the quantification performed by HPAEC-PAD. In addition, we show that simple boiling is not sufficient to inactivate LPMOs and we suggest to apply a metal chelator in addition to
\end{abstract}

M. B. Keller · C. Felby · B. M. Blossom ( $₫)$

Department of Geosciences and Natural Resource

Management, University of Copenhagen, Copenhagen, Denmark

e-mail: kbm@ign.ku.dk

\section{A. Labate}

Luiz de Queiroz College of Agriculture, University of São Paulo, Piracicaba, Brazil

\section{O. A. Pellegrini · P. Higasi · I. Polikarpov}

São Carlos Institute of Physics, University of São Paulo,

São Carlos, Brazil

\section{R. K. Singh}

Department of Chemistry, University of Copenhagen,

Copenhagen, Denmark boiling or to drastically increase $\mathrm{pH}$ for proper inactivation.

Conclusions We conclude that the versatility of this simple enzymatic assay makes it useful in a wide range of experiments in basic and applied LPMO research and without the need for expensive instrumentation, e.g. HPAEC-PAD.

Keywords Lytic polysaccharide monooxygenase (LPMO) - Biomass degradation · Quantification assay $\cdot$ LPMO inactivation $\cdot$ Cellulose oxidation · Gluconic acid

\section{Introduction}

Since their discovery, Lytic polysaccharide monooxygenases (LPMOs) have received considerable interest, due to their ability to boost the degradation of lignocellulose (Vaaje-Kolstad et al. 2010; Horn et al. 2012). LPMOs are mono-copper enzymes with the active site positioned on a relatively flat surface enabling them to interact with crystalline polysaccharides. The catalytic mechanism of LPMOs involves the reduction of the copper from $\mathrm{Cu}$ (II) to $\mathrm{Cu}$ (I) by an external reducing agent. Hereafter, the copper-active site of the LPMO reacts with either $\mathrm{O}_{2}$ or $\mathrm{H}_{2} \mathrm{O}_{2}$ (Walton and Davies 2016; Bissaro et al. 2017) and binds an activated oxygen species that enables the 
oxidation of the polysaccharide substrate. The oxidative cleavage of the $\beta-1,4$ glycosidic bonds can occur at the $\mathrm{C} 1$ or the $\mathrm{C} 4$ position or both, generating oxidized products, both soluble and insoluble (Quinlan et al. 2011). The soluble products are commonly detected by anion exchange chromatography coupled to a pulsed amperometric detector (HPAEC-PAD) (Westereng et al. 2013). It offers high sensitivity and provides detailed information about the product pattern but its limitations are the absence of commercially available reference compounds, time-consuming sample analysis, and costly instrumentation.

LPMO assays have been developed to circumvent some of these challenges. The Amplex red assay provides a fast measure of LPMO reactivity and is based on the release of $\mathrm{H}_{2} \mathrm{O}_{2}$ in the absence of a substrate (Kittl et al. 2012). Recently, a spectrophotometrical assay was developed that exploits peroxidase activity of some LPMOs to oxidize 2,6dimethoxyphenol (Breslmayr et al. 2018). While both assays can be employed to detect LPMO reactivity, the results cannot be directly correlated to the oxidative activity of LPMOs on polysaccharides. Soluble LPMO products have been analyzed with by carbohydrate gel electrophoresis (PACE) (Quinlan et al. 2011; Frandsen et al. 2016) yielding semi-quantitative results. LPMO activity has also been analyzed using chromogenic polysaccharide hydrogel substrates (Kračun et al. 2015). Assays for quantifying C1-oxidized moieties in the insoluble fraction of the LPMO on pure cellulose model substrates were described, such as a fluorescence-based approach (Vuong et al. 2017), and a colorimetric cation-based assay (Wang et al. 2018). Despite the value of these methods, LPMO activity assays and especially methods that can simplify the quantification of LPMO oxidation, without the necessity of expensive specialized instrumentation, not only on cellulosic model substrates but on complex substrates, such as lignocellulosic biomass are still lacking.

We describe a simple quantification method for cellulose active C1-oxidizing LPMOs, employing a commercially available glycoside hydrolase to hydrolyze the oxidation products to yield gluconic acid and glucose. Subsequently, gluconic acid is quantified spectrophotometrically which is suitable for microplate formats. The release of $\mathrm{C} 1$-oxidized products by $T t$ LPMO9E on microcrystalline cellulose (Avicel) and steam pretreated wheat straw (PWS) was quantified and compared to the results by HPEAC-PAD.

\section{Methods}

Substrates

The activity of Thielavia terrestris LPMO9E ( $T t$ LPMO9E) (previously $T t \mathrm{GH} 61 \mathrm{E}$ ) was determined on the two substrates; Avicel PH101 (Sigma-Aldrich), and pretreated wheat straw (PWS). The wheat straw was hydrothermally pretreated at $190{ }^{\circ} \mathrm{C}$ for $10 \mathrm{~min}$ at an initial solid loading of $35 \%$. The heating was done by injection of steam, decreasing the solids level to $15 \%$ after 10 min treatment. Using standard NREL methods the composition was determined to be $54 \%$ glucan, 4\% xylan, 34\% lignin, and 6\% ash. The substrates were washed five times in MilliQ water and twice in $50 \mathrm{mM}$ citrate buffer $\mathrm{pH} 6$ to remove soluble sugars prior to the incubation with $T t$ LPMO9E.

\section{Enzymes}

$T t$ LPMO9E was donated by Novozymes A/S (Bagsværd, Denmark). The TtLPMO9E concentration was determined by absorbance at $280 \mathrm{~nm}$ using a molar extinction coefficient of $58,120 \mathrm{M}^{-1} \mathrm{~cm}^{-1}$. Novozym 188 was obtained from Novozymes A/S (Bagsværd, Denmark). $\beta$-glucosidase from Aspergillus niger and cellobiohydrolase I (CBHI) from Trichoderma longibrachiatum were purchased from Megazyme with a specified activity of $40 \mathrm{U} \mathrm{ml}^{-1}$ and a concentration of $10 \mathrm{mg} \mathrm{ml}^{-1}$, respectively.

Preparation of C1-oxidized cello-oligosaccharide standards for the HPAEC-PAD

C1-oxidized cello-oligosaccharide standards for the HPAEC-PAD were prepared by oxidizing pure cellooligosaccharides (Megazyme) with iodine as earlier described for the preparation of cellobionic acid (Green et al. 1976; Hildebrand et al. 2016), with the following modifications:

The cello-oligosaccharide was dissolved in a minimum of deionized water and heated at $55^{\circ} \mathrm{C}$ for 10 min until dissolved. A solution of iodine in methanol was added to a final molar ratio of 1:2:110 (cello-oligosaccharide:iodine:methanol) and the 
solution was heated at $40{ }^{\circ} \mathrm{C}$ for $15 \mathrm{~min}$. At room temperature $4 \%(\mathrm{w} / \mathrm{v})$ potassium hydroxide-methanol solution was slowly added to the solution and left for an additional $30 \mathrm{~min}$ to a final molar ratio of 1:12 (cello-oligosaccharide:potassium hydroxide). $20 \mathrm{~mL}$ deionized water was added to the solution and the methanol was gently boiled off in a rotary evaporator. The $\mathrm{pH}$ of the solution was set to $\mathrm{pH} 5$ with citric acid.

All procedures were done with constant stirring.

\section{Inactivation of $T t \mathrm{LPMO} 9 \mathrm{E}$}

Samples of Avicel $50 \mathrm{~g} \mathrm{~L}^{-1}, 50 \mathrm{mM}$ citrate buffer $\mathrm{pH}$ 6, $1 \mu \mathrm{M} T t \mathrm{LPMO}$, and $2 \mathrm{mM}$ ascorbic acid in a final volume of $200 \mu \mathrm{l}$ were mixed in Eppendorf tubes. The samples were treated with or without $1 \mathrm{mM}$ EDTA addition, with or without $50 \mathrm{mM} \mathrm{NaOH}$ and with or without incubation in a water bath at $100{ }^{\circ} \mathrm{C}$ for 20 min. A positive control, not treated with EDTA, $\mathrm{NaOH}$, or heat, was included. The samples were incubated in an Eppendorf ThermoMixer $\mathrm{C}$ at $50{ }^{\circ} \mathrm{C}$ for $6 \mathrm{~h}$ at $1100 \mathrm{rpm}$. Subsequently, the samples were centrifuged and the supernatant was kept for measuring $\mathrm{C} 1$-oxidized sites as described below.

\section{TtLPMO9E activity}

Samples of Avicel $\left(50 \mathrm{~g} \mathrm{~L}^{-1}\right)$ or PWS $\left(20 \mathrm{~g} \mathrm{~L}^{-1}\right)$, $1 \mu \mathrm{M} T t \mathrm{LPMO} 9 \mathrm{E}, 2 \mathrm{mM}$ ascorbic acid in $50 \mathrm{mM}$ citrate buffer $\mathrm{pH} 6$ in a final volume of $200 \mu$ l were incubated in an Eppendorf ThermoMixer $\mathrm{C}$ at $50{ }^{\circ} \mathrm{C}$. At varying time points $(0-24 \mathrm{~h})$, the enzymatic reaction was quenched by the addition of $\mathrm{NaOH}$ to a final concentration of $50 \mathrm{mM}$.

After $24 \mathrm{~h}$, the samples were centrifuged at $1800 \times g$ and the supernatant was used for measuring C1-oxidized sites as described below.

The pellet was further hydrolyzed for measuring $\mathrm{C} 1$-oxidized sites in the insoluble fraction. The pellet was washed five times in $200 \mu \mathrm{L} 50 \mathrm{mM}$ citrate buffer $\mathrm{pH}$ 5, by centrifugation and resuspension, to decrease $\mathrm{pH}$ and remove soluble sugars. The pellet was resuspended in $50 \mathrm{mM}$ citrate buffer. Cellobiohydrolase I (CBHI) from Trichoderma longibrachiatum was added to a final concentration of $0.5 \mathrm{~g} \mathrm{~L}^{-1}$ in a final volume of $200 \mu \mathrm{L}$. The samples were incubated in an Eppendorf ThermoMixer $\mathrm{C}$ at $50{ }^{\circ} \mathrm{C}$ for $24 \mathrm{~h}$ at $1100 \mathrm{rpm}$ after which the samples were centrifuged.
The supernatant was used for measuring C1-oxidized sites revealed by the CBHI as described below.

Quantification of gluconic acid by HPAEC-PAD

$100 \mu \mathrm{L}$ of the supernatant was diluted in $50 \mathrm{mM}$ citrate buffer $\mathrm{pH} 5$ together with $5 \mathrm{U} \mathrm{ml}^{-1} \beta$ glucosidase from Aspergillus niger to a final volume of $200 \mu \mathrm{L}$. The samples were incubated at $50{ }^{\circ} \mathrm{C}$ for $24 \mathrm{~h}$ before the samples were filtered through a nylon filter with a pore size of $0.45 \mu \mathrm{m}$ and loaded in HPLC vials.

The samples were analyzed by high-performance anion-exchange chromatography (HPAEC) performed on an ICS5000 system, equipped with a pulsed amperometric detector (PAD) (Thermo Scientific) with an analytical CarboPac PA1 column $(2 \times 250)$ as described elsewhere (Westereng et al. 2013) with some modifications: The analytes were eluted at $0.25 \mathrm{~mL} \mathrm{~min}^{-1}$ at $30{ }^{\circ} \mathrm{C}$ and the initial conditions were $100 \%$ eluent $\mathrm{A}(0.1 \mathrm{M} \mathrm{NaOH})$. A linear gradient was applied increasing the proportion of eluent B (1 M $\mathrm{NaOAc}$ in $0.1 \mathrm{M} \mathrm{NaOH}$ ) to $90 \% \mathrm{~A}: 10 \% \mathrm{~B}$ after $10 \mathrm{~min}$. An exponential gradient (curve 6) was applied to reach $83.1 \% \mathrm{~A}: 16.9 \% \mathrm{~B}$ after $22 \mathrm{~min}$ and $0 \%$ A:100\% B after $23 \mathrm{~min}$. These conditions were kept for $4 \mathrm{~min}$. The column was reconditioned by running initial conditions for $15 \mathrm{~min}$. Gluconic acid was quantified based on external standards of $0-250 \mathrm{mg}$ $\mathrm{L}^{-1}$ gluconic acid in $50 \mathrm{mM}$ citrate $\mathrm{pH} 5$.

Quantification of gluconic acid by the D-Gluconic acid/D-Glucono- $\delta$-lactone assay kit

Gluconic acid was quantified using the assay kit from Megazyme (Bergmeyer and Moellering 1988). The method is based on phosphorylation of gluconic acid to D-gluconate-6-phosphate by ATP in the presence of gluconate kinase with the simultaneous formation of ADP. In the presence of NADP, gluconate-6-phosphate is oxidatively decarboxylated by 6-phosphogluconate dehydrogenase to ribulose-5-phosphate with the stoichiometrically formation of NADPH. The assay was performed according to the microplate procedure described by the manufacturer with the following modifications: $50 \mu \mathrm{L}$ of the supernatant was mixed with $160 \mu \mathrm{L}$ MilliQ water, $20 \mu \mathrm{L}$ solution I (buffer), $20 \mu \mathrm{L}$ solution II (NADP + ATP), $6 \mu \mathrm{L}$ solution III (6-PGDH) diluted three times in MilliQ 
water. The samples were mixed in an Eppendorf ThermoMixer for $4 \mathrm{~min}$ at $600 \mathrm{rpm}$ after which the absorbance was measured at $340 \mathrm{~mm}$ in microplate reader (Molecular Devices SpectraMax i3). $6 \mu \mathrm{L}$ solution IV (GCK) diluted three times with MilliQ water was added to the samples and the plate was mixed in an Eppendorf ThermoMixer at $600 \mathrm{rpm}$ for $6 \mathrm{~min}$. The absorbance was measured at $340 \mathrm{~nm}$ and the difference was used to quantify gluconic acid based on external standards of $0-250 \mathrm{mg} \mathrm{L}^{-1}$ gluconic acid in $50 \mathrm{mM}$ citrate $\mathrm{pH} 5$. According to the supplier's information, the range of linearity of the UV-signal is $0.1-5 \mu \mathrm{g}$ gluconic acid per sample in a sample volume of $1-200 \mu \mathrm{L}$. Using the guidelines of the International Conference on Harmonisation (ICH), we calculated the quantitation limit (LOQ) as LOQ = $10 \mathrm{Sa} / \mathrm{b}$, where $\mathrm{Sa}$ is the standard error of the $y$-intercept of the regression line of the gluconic acid standard curve and $\mathrm{b}$ is the slope of the regression line of the gluconic acid standard curve. Using this approach, we found that $\mathrm{LOQ}=0.27 \mathrm{mg}$ gluconic acid $\mathrm{L}^{-1}$.

\section{Quantification of glucose}

The hydrolysis yield was determined on an Ultimate 3000 high-performance liquid chromatography equipped with an ultraviolet detector at $210 \mathrm{~nm}$ (Thermo Scientific). The separation was performed on a Phenomenex Rezex ROA column at $80{ }^{\circ} \mathrm{C}$ with a mobile phase of $5 \mathrm{mM} \mathrm{H} \mathrm{H}_{2}$ at a flow rate of $0.6 \mathrm{~mL} \mathrm{~min}^{-1}$. The glucose was quantified based on external standards of $0-10 \mathrm{~g} \mathrm{~L}^{-1}$ glucose in $50 \mathrm{mM}$ citrate $\mathrm{pH} 5$.

\section{Results and discussion}

Hydrolysis of oxidized products

To decrease product complexity, the products of LPMO-catalysis are often hydrolyzed by glycoside hydrolases prior to the quantification by HPAEC-PAD (Cannella et al. 2016; Bissaro et al. 2017; Frommhagen et al. 2017). Here, we assessed the applicability of two commercially available $\beta$-glucosidases, Novozym 188 and $\beta$-glucosidase from Aspergillus niger to hydrolyze the soluble products generated by
$T t$ LPMO9E from a cellulosic substrate to the monomers, glucose and gluconic acid (Fig. 1).

The lowest $\beta$-glucosidase concentration capable of hydrolyzing the $T t \mathrm{LPMO}$ E products generated in the following experiments to glucose and gluconic acid was determined to be $1.25 \mathrm{U} / \mathrm{ml}$ A. niger $\beta$-glucosidase corresponding to a specific activity on 4-Nitrophenyl $\beta$-D-glucuronide of $1.26 \times 10^{-3} \mu \mathrm{mol}$ p-nitrophenol $\mathrm{min}^{-1}$ and Novozym 188 corresponding to a specific activity of $1.16 \times 10^{-3} \mu \mathrm{mol}$ p-nitrophenol $\mathrm{min}^{-1}$ according to the $\beta$-glucosidase activity assay described elsewhere (Gunata et al. 1990). These concentrations were used throughout the work presented here. The product profiles were analyzed by HPAEC-PAD (Fig. 1).

Both $\beta$-glucosidases hydrolyzed the TtLPMO9E products to the monomers, glucose and gluconic acid (GlcA). As the Novozym 188 contained impurities eluting together with glucose and gluconic acid, the $A$. niger $\beta$-glucosidase was selected for further use.

\section{Inactivation of $T t \mathrm{LPMO} 9 \mathrm{E}$}

As $T t$ LPMO9E does not have activity on soluble oligosaccharides (Westereng et al. 2015), the reaction in the supernatant can simply be stopped by filtration. Efficient inactivation of the LPMOs is, however, essential to quantify the activity in the insoluble fraction. Prior to the hydrolysis of the LPMO products by glucoside hydrolases, common procedures to quench the LPMO reaction are $10 \mathrm{~min}$ incubations at $100{ }^{\circ} \mathrm{C}$ (Cannella et al. 2016; Frommhagen et al. 2017) or by increasing pH (Isaksen et al. 2014; Bissaro et al. 2017). Previous studies have shown that chelating agents, such as EDTA can remove the copper atom from the LPMO active site (VaajeKolstad et al. 2010; Kracher et al. 2017), suggesting that the addition of EDTA could effectively inactivate LPMOs.

The inactivation efficiencies were tested by measuring the activity of $T t$ LPMO9E after boiling for 20 min with and without the addition of EDTA or after the addition of sodium hydroxide, and their activity was compared to the activity of a non-treated TtLPMO9E samples (Fig. 2).

$T t$ LPMO9E retained approximately $50 \%$ of its activity after boiling for $20 \mathrm{~min}$. This may be due to reversible thermal unfolding as has previously been reported for NcLPMO9C and TaLPMO9A, 


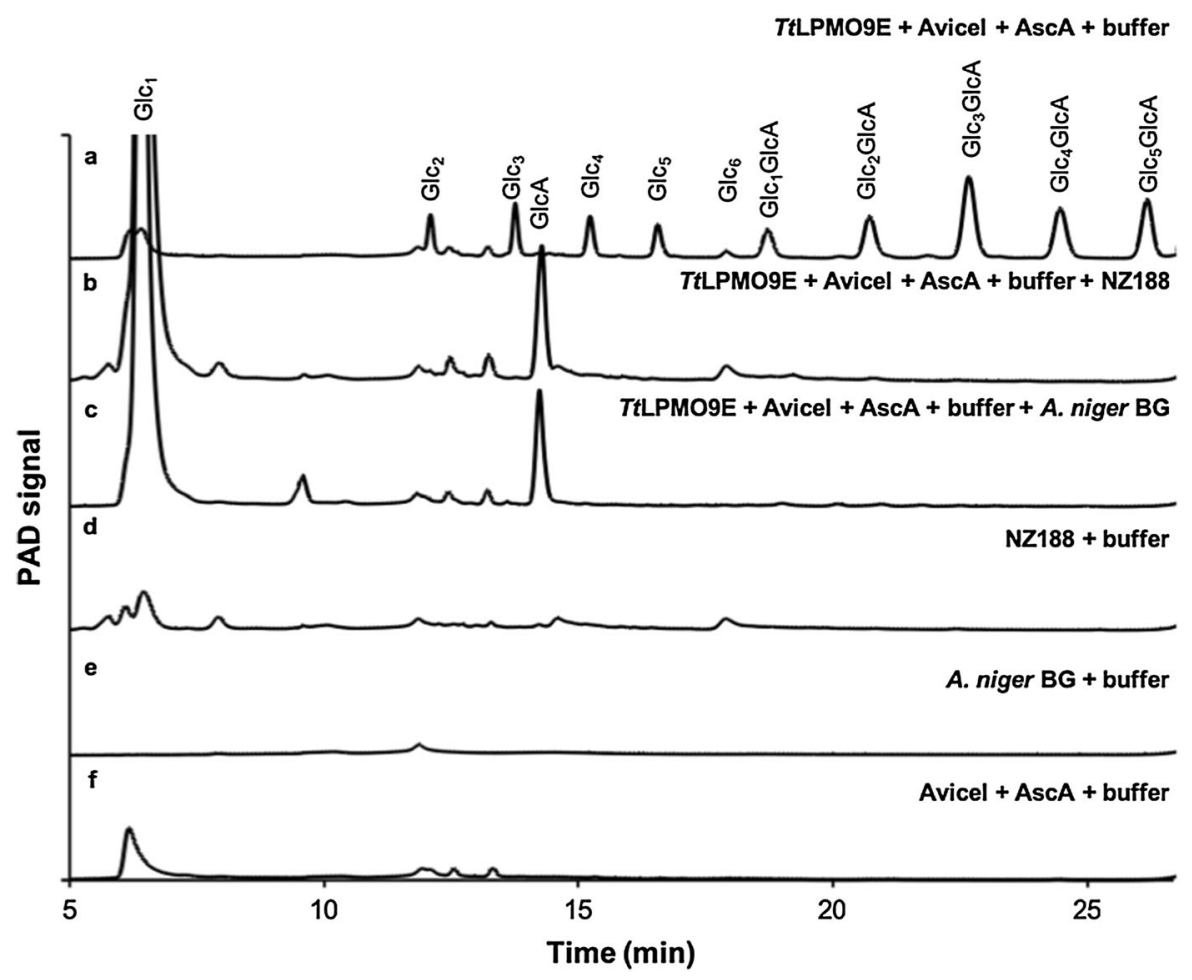

Fig. 1 HPAEC-PAD chromatograms and elution patterns. a, The supernatant of $50 \mathrm{~g} \mathrm{~L}^{-1}$ Avicel incubated with $1 \mu \mathrm{M}$ $T t \mathrm{LPMO} 9 \mathrm{E}$ and $2 \mathrm{mM}$ ascorbic acid in $50 \mathrm{mM}$ citrate buffer $\mathrm{pH}$ 6 for $6 \mathrm{~h}$ at $50{ }^{\circ} \mathrm{C}$. The peaks are assigned based on external standards of C1-oxidized cello-oligosaccharide standards (not shown). The oligosaccharides are abbreviated $\mathrm{Glc}_{\mathrm{N}}$ and

respectively (Kracher et al. 2017; Singh et al. 2019). These findings suggest that if boiling is used to attempt inactivation of LPMOs, it should be followed by careful evaluation of residual activity. The addition of EDTA decreased the activity by approximately $50 \%$. A combination of boiling and addition of EDTA decreased the activity of $T t \mathrm{LPMO}$ E to less than $10 \%$ compared to the positive control not treated with heat or EDTA. A similar decrease in activity was obtained by the addition of $50 \mathrm{mM} \mathrm{NaOH}$ to reach $\mathrm{pH} 11$.

\section{Quantification of the LPMO reaction}

The activity of TtLPMO9E was measured by quantifying the $\mathrm{C} 1$-oxidation products in the soluble and the insoluble fraction of microcrystalline cellulose (Avicel) and pretreated wheat straw (PWS) (Fig. 3). The results should not be interpreted as the absolute quantification of insoluble products, as only a fraction of the insoluble products was hydrolyzed.
$\mathrm{Glc}_{\mathrm{N}} \mathrm{GlcA}$ with $\mathrm{N}$ being the number of glucose units and GlcA being gluconic acid. $\mathbf{b}$, The supernatant from $\mathbf{a}$ incubated with Novozym 188. c, The supernatant from a incubated with $A$. niger $\beta$-glucosidase. d, Novozym 188. e, A. niger $\beta$-glucosidase. f The supernatant of $50 \mathrm{~g} \mathrm{~L}^{-1}$ Avicel incubated with $2 \mathrm{mM}$ ascorbic acid for $6 \mathrm{~h}$ at $50^{\circ} \mathrm{C}$

The formation of gluconic acid (GlcA) in the soluble fraction of Avicel incubated with $T t$ LPMO9E and ascorbic acid increased up to approximately $175 \mathrm{mg} \mathrm{L}^{-1}$ after 0.5 to $6 \mathrm{~h}$. Between 6 and $24 \mathrm{~h}$, no further changes in GlcA concentration were detected in the soluble fraction. GlcA formation in the insoluble fraction of Avicel increased up to approximately $35 \mathrm{mg} \mathrm{L} \mathrm{L}^{-1}$ after $24 \mathrm{~h}$. Interestingly, after $6 \mathrm{~h}$ of incubation an increase in GlcA was solely detected in the insoluble fraction whereas no increase in GlcA was detected in the soluble fraction.

For the reactions on pretreated wheat straw, the GlcA content in the soluble fraction increased from 0.5 to $6 \mathrm{~h}$ up to approximately $175 \mathrm{mg} \mathrm{L}^{-1}$. As with the reaction on Avicel, the GlcA concentration in the soluble fraction remained unchanged between 6 and $24 \mathrm{~h}$. In the insoluble fraction, the formation of GlcA increased steadily up to approximately $60 \mathrm{mg} \mathrm{L}^{-1}$ after $24 \mathrm{~h}$. 


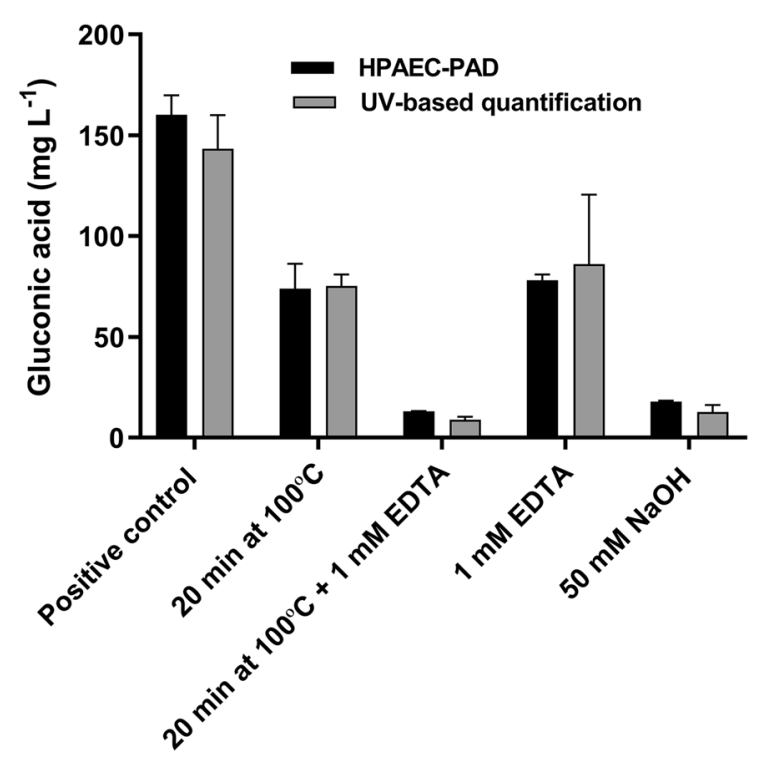

Fig. 2 Evaluation of methods to inactivate $T t \mathrm{LPMO}$ E by quantification of gluconic acid. $50 \mathrm{~g} \mathrm{~L}^{-1}$ Avicel were incubated with $2 \mathrm{mM}$ ascorbic acid and $1 \mu \mathrm{M} T t$ LPMO9E in $50 \mathrm{mM}$ citrate buffer $\mathrm{pH} 6$ for $6 \mathrm{~h}$ at $50{ }^{\circ} \mathrm{C}$. Prior to the incubation, the samples were treated with either, $20 \mathrm{~min}$ incubation at $100{ }^{\circ} \mathrm{C}$, 20 min incubation at $100^{\circ} \mathrm{C}+1 \mathrm{mM}$ EDTA, $1 \mathrm{mM}$ EDTA, or $50 \mathrm{mM} \mathrm{NaOH}$. A positive control, not treated with $\mathrm{NaOH}$, EDTA, or incubation at $100{ }^{\circ} \mathrm{C}$, was included. Gluconic acid was quantified by HPAEC-PAD (black bars) and by the UVbased gluconic acid kit (grey bars). All incubations were performed in triplicate and the standard deviations of the samples are represented by error bars

The UV-based enzymatic GlcA quantification method produced similar results and detection limits as the HPAEC-PAD when analyzing products from the soluble and insoluble fractions of Avicel as well as PWS. The results of the two methods showed a linear correlation with $\mathrm{r}^{2}$ values within the four data groups varying between 0.986 and 0.996 .

Furthermore, dose-response curves of enzyme titration (Fig. 4a) and substrate loading (Fig. 4b) were performed on PWS and the results were fitted to a hyperbolic function with the best fit of $\left[y=197.97^{*} \times\right.$ ] $(2.51+\mathrm{x})]$ for the enzyme titration data. Likewise, the dose-response curve with increased substrate load was fitted to a hyperbolic Michalies-Menten like function,

${ }_{p} V_{s s}=\frac{{ }_{p} V_{\max } S_{0}}{{ }_{p} K_{M}+S_{0}}$

as described for other interfacial enzymes (CruysBagger et al. 2013) with $\mathrm{V}_{\mathrm{ss}}$ being the specific activity
Fig. 3 Quantification of the soluble and insoluble products from microcrystalline cellulose and pretreated wheat straw (PWS). $50 \mathrm{~g} \mathrm{~L}^{-1}$ Avicel or $20 \mathrm{~g} \mathrm{~L}^{-1}$ PWS incubated with $2 \mathrm{mM}$ ascorbic acid and $1 \mu \mathrm{M}$ TtLPMO9E in $50 \mathrm{mM}$ citrate buffer $\mathrm{pH}$ 6 at $50{ }^{\circ} \mathrm{C}$ from 0 to $24 \mathrm{~h}$. a, Supernatant of Avicel incubated with $T t$ LPMO9E and ascorbic acid incubated with $5 \mathrm{U} \mathrm{mL}^{-1} A$. niger $\beta$-glucosidase. b, Pellet of Avicel incubated with $T t$ LPMO9E and ascorbic acid incubated with $0.5 \mathrm{~g} \mathrm{~L}^{-1} \mathrm{~T}$. longibrachiatum $\mathrm{CBHI}$ and $5 \mathrm{U} \mathrm{mL}^{-1}$ A. niger $\beta$-glucosidase. c, Supernatant of PWS incubated with TtLPMO9E and ascorbic acid incubated with $5 \mathrm{U} \mathrm{mL}^{-1} A$. niger $\beta$-glucosidase. d, Pellet of PWS incubated with $T t \mathrm{LPMO} 9 \mathrm{E}$ and ascorbic acid incubated with $0.5 \mathrm{~g} \mathrm{~L}^{-1} \mathrm{~T}$. longibrachiatum $\mathrm{CBHI}$ and $5 \mathrm{U} \mathrm{mL}^{-1} A$. niger $\beta$-glucosidase. Gluconic acid detected after $0 \mathrm{~h}$ of incubation was subtracted from the results. Gluconic acid was quantified by HPAEC-PAD (black bars) and by the UV-based gluconic acid kit (grey bars). All results are averages of triplicate measurements. The standard deviations of the samples are represented by error bars. e and $\mathbf{f}$, Correlation between the quantification results measured by HPAEC-PAD (y-axis) and gluconic acid kit (x-axis) from a-d. The equation for the best linear regression (slope $=0.9$ ) and the coefficient of determination, $r^{2}\left(r^{2}=0.98\right)$ is shown. $f$ : Enlargement of the correlation at gluconic acid concentrations from 0 to $50 \mathrm{mg} \mathrm{L}^{-1}$. For clarity, standard deviations are not shown

(product/time/enzyme concentration), ${ }_{\mathrm{p}} \mathrm{V}_{\text {max }}$ being the maximal specific activity, $\mathrm{S}_{0}$ being the substrate load, and ${ }_{\mathrm{p}} \mathrm{K}_{\mathrm{M}}$ the Michaelis-Menten constant. Using the enzymatic assay, the ${ }_{\mathrm{p}} \mathrm{K}_{\mathrm{M}}$ and the ${ }_{\mathrm{p}} \mathrm{V}_{\text {max }} / \mathrm{E}_{0}$ were derived from the regression analysis to be $49.80 \mathrm{~g} \mathrm{~L}^{-1}$ and $0.064 \mathrm{~s}^{-1}\left(3.8 \mathrm{~min}^{-1}\right)$, respectively. The ladder being in line with the results given in a recent review, comparing the apparent enzyme rates of LPMOs, that showed that the apparent catalytic rates $\left(\mathrm{k}_{\mathrm{cat}}\right)$ of LPMOs fall in a relatively small range roughly between 1 and $10 \mathrm{~min}^{-1}$ when not considering $\mathrm{H}_{2} \mathrm{O}_{2}$ or light-driven reactions (Bissaro et al. 2018).

\section{Conclusions}

We have described a simple, fast, and sensitive UVbased enzymatic assay to quantify specifically the $\mathrm{C} 1$ oxidation of cellulose active LPMOs. In recent years, a number of LPMO assays have been developed to decrease the analysis time compared to HPAEC-PAD, as well as eliminating the need for oxidized oligosaccharide standards that are not commercially available, and expensive instrumentation. These methods all have distinct advantages making them suitable for different applications. The method reported in this 

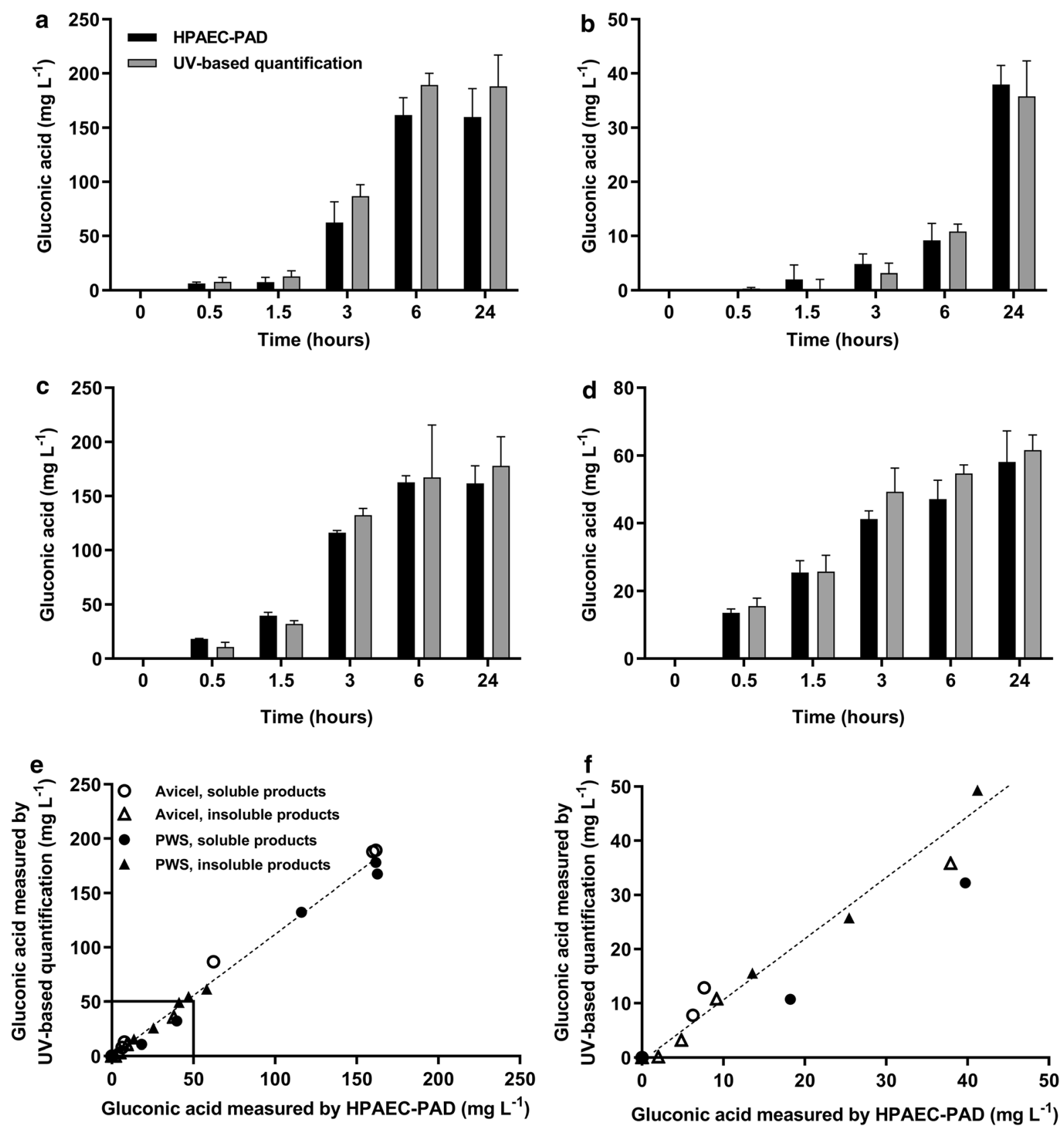

paper excels by its simplicity and its ability to quantify soluble and insoluble LPMO products on a pure cellulose substrate as well as on a lignocellulosic biomass. The UV-based enzymatic assay is suitable for an auto-analyzer format and, with an analysis time of approximately 30 min per 96 samples in a microtiter plate, compared to approximately $15 \mathrm{~min}$ per sample or $24 \mathrm{~h}$ per 96 samples in a microtiter plate with
HPAEC, the method offers fast measurements (Fig. 5).

Furthermore, we compared methods for TtLPMO9E inactivation and demonstrated that a boiling is not sufficient. Thus, we suggest to apply a metal chelator while boiling or to drastically increase $\mathrm{pH}$ for proper inactivation. 


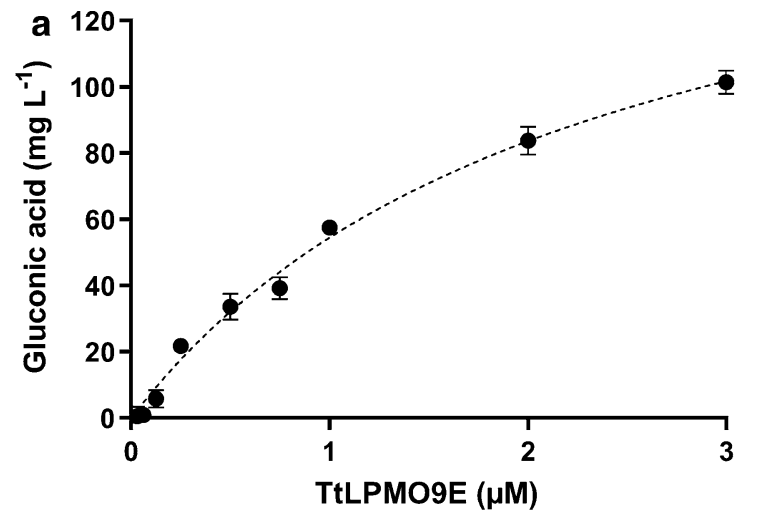

Fig. 4 Dose-response curves of enzyme and substrate titrations. a, Quantification of the soluble products of $20 \mathrm{~g} \mathrm{~L}^{-1} \mathrm{PWS}$ incubated with $2 \mathrm{mM}$ ascorbic acid and varying concentrations of $T t$ LPMO9E in $50 \mathrm{mM}$ citrate buffer $\mathrm{pH} 6$ at $50{ }^{\circ} \mathrm{C}$ for $3 \mathrm{~h}$. The dotted line represents the best fit of a hyperbolic function. $\mathbf{b}$, specific activity of TtLPMO9E on PWS measured by

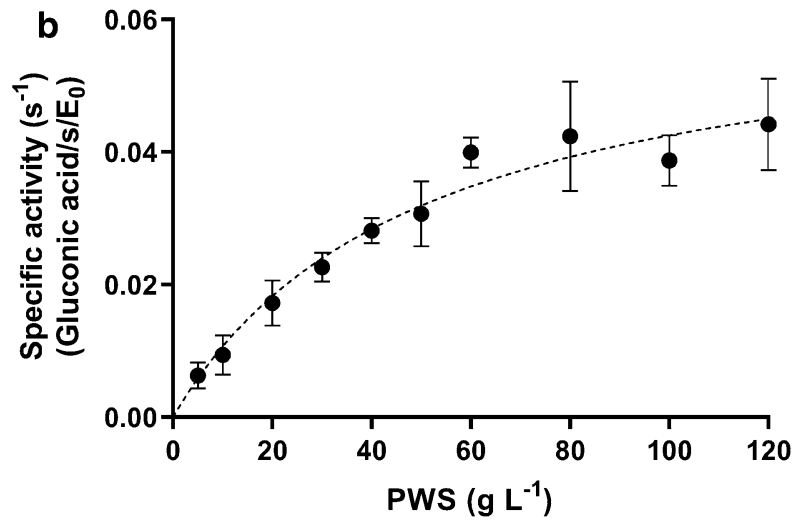

quantifying the gluconic acid in the soluble products of varying loads of PWS incubated with $2 \mathrm{mM}$ ascorbic acid and $0.25 \mu \mathrm{M}$ $T t \mathrm{LPMO} 9 \mathrm{E}$ in $50 \mathrm{mM}$ citrate buffer $\mathrm{pH} 6$ at $50{ }^{\circ} \mathrm{C}$ in $3 \mathrm{~h}$. The dotted line represents the best fit of a hyperbolic function. All results are averages of triplicate measurements. The standard deviations of the samples are represented by error bars

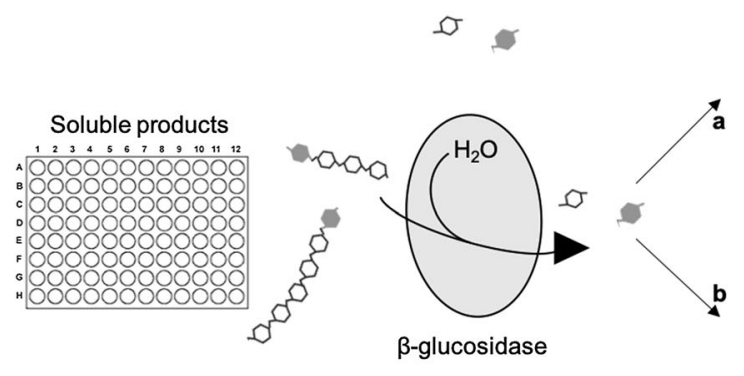

Fig. 5 Schematic representation of the UV-based enzymatic assay to quantify LPMO activity on cellulose. After the LPMO reaction, the plates were centrifuged and the soluble products were collected in the supernatant. $\beta$-glucosidase was added to the soluble products and the plates were incubated at $50{ }^{\circ} \mathrm{C}$ overnight after which gluconic acid was quantified by HPAECPAD (a) and by the UV-based enzymatic assay (b). a: The samples were filtered and gluconic acid was quantified on a CarboPac PA1 column $(2 \times 250)$ on a HPAEC-PAD. The peak areas were used for the quantification of gluconic acid based on

The quantification method may be useful in other experiments, such as for comparison of activities of different C1-oxidizing cellulose active LPMOs, for studies of LPMO activities on different substrates and for testing compounds that might influence LPMO activity. Such experiments are technically straightforward by using the quantification approach outlined here, but changing conditions should be followed by

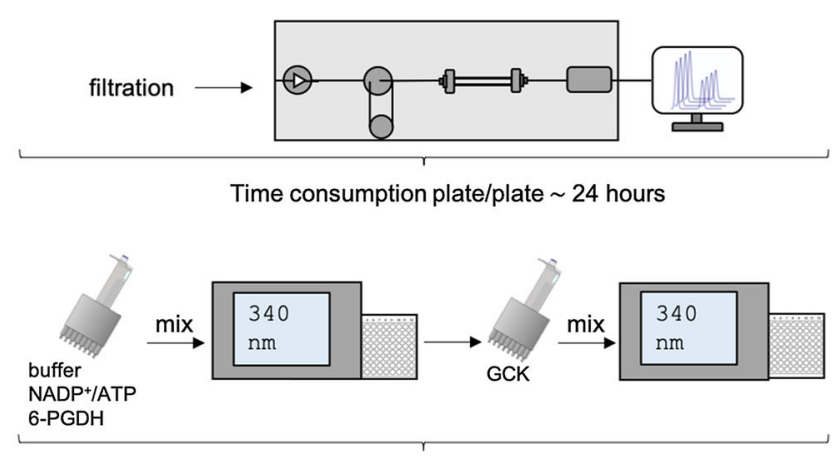

Time consumption plate/plate $\sim 30 \mathrm{~min}$

external standards. The time consumption was 30 min per plate, however this could be optimized for shorter runs. b: Buffer, $\mathrm{NADPH}+$, and 6-Phosphoglucanate dehydrogenase was added to the samples. The samples were mixed for $4 \mathrm{~min}$ in a thermomixer at $600 \mathrm{rpm}$ and the absorbance was measured at $340 \mathrm{~nm}$ after which gluconate kinase was added to the samples and the plates were mixed for $6 \mathrm{~min}$ in a thermomixer at $600 \mathrm{rpm}$ and the absorbance was measured at $340 \mathrm{~nm}$. The difference in absorbance was used for the quantification of gluconic acid based on external standards

calibration against an alternative quantification technique to exclude potential interferences.

Acknowledgements We would like to sincerely thank Anne Stenbæk and Johan Pelck Olsen from Novozymes A/S for their support and supply of enzyme.

Funding This work was funded by the Innovation Found, Denmark 7043-00016B (ENACT), the Novo Nordisk Foundation grant NNF16OC0021832 and by Fundação de 
Amparo à Pesquisa do Estado de São Paulo (FAPESP, Brazil) via Grants 2015/13684-0 and 2017/01165-4.

\section{Compliance with ethical standards}

Conflict of interest All authors declare that they have no conflicts of interest with the contents of this article.

Research involving human and animal rights This article does not contain any studies with human participants or animals performed by any of the authors.

Open Access This article is distributed under the terms of the Creative Commons Attribution 4.0 International License (http:// creativecommons.org/licenses/by/4.0/), which permits unrestricted use, distribution, and reproduction in any medium, provided you give appropriate credit to the original author(s) and the source, provide a link to the Creative Commons license, and indicate if changes were made.

\section{References}

Bergmeyer HU, Moellering H (1988) D-Gluconate (D-Glucono $\delta$-lactone) and D-Gluconate 6-Phosphate; Methods in enzymatic analysis, 3rd edn. VCH Publishers (UK) Ltd., Cambrigde, p 220-227

Bissaro B, Røhr ÅK, Müller G et al (2017) Oxidative cleavage of polysaccharides by monocopper enzymes depends on $\mathrm{H}_{2} \mathrm{O}_{2}$. Nat Chem Biol. https://doi.org/10.1038/nchembio. 2470

Bissaro B, Várnai A, Røhr ÅK, Eijsink VGH (2018) Oxidoreductases and reactive oxygen species in conversion of lignocellulosic biomass. Microbiol Mol Biol Rev 82:e00029-e118. https://doi.org/10.1128/MMBR.0002918

Breslmayr E, Hanžek M, Hanrahan A et al (2018) A fast and sensitive activity assay for lytic polysaccharide monooxygenase. Biotechnol Biofuels 11:79. https://doi. org/10.1186/s13068-018-1063-6

Cannella D, Möllers KB, Frigaard N-UU et al (2016) Lightdriven oxidation of polysaccharides by photosynthetic pigments and a metalloenzyme. Nat Commun 7:1134. https://doi.org/10.1038/ncomms11134

Cruys-Bagger N, Elmerdahl J, Praestgaard E et al (2013) A steady-state theory for processive cellulases. FEBS J 280:3952-3961. https://doi.org/10.1111/febs.12397

Frandsen KEH, Simmons TJ, Dupree P et al (2016) The molecular basis of polysaccharide cleavage by lytic polysaccharide monooxygenases Europe PMC Funders Group. Nat Chem Biol 12:298-303. https://doi.org/10. 1038/nchembio.2029

Frommhagen M, Westphal AH, Hilgers R et al (2017) Quantification of the catalytic performance of $\mathrm{C} 1$-cellulosespecific lytic polysaccharide monooxygenases. Appl Microbiol Biotechnol https://doi.org/10.1007/s00253-0178541-9

Green JW, Thompson NS, Pearl IA (1976) Study of the carbohydrate peeling and stopping reactions under the conditions of oxygen-alkali pulping. Appleton, Wisconsin
Gunata YZ, Bayonove CL, Cordonnier RE et al (1990) Hydrolysis of grape monoterpenyl glycosides by Candida molischiana and Candida wickerhamii $\beta$-glucosidases. J Sci Food Agric 50:499-506. https://doi.org/10.1002/jsfa. 2740500408

Hildebrand A, Addison JB, Kasuga T, Fan Z (2016) Cellobionic acid inhibition of cellobiohydrolase I and cellobiose dehydrogenase. Biochem Eng J 109:236-242. https://doi. org/10.1016/j.bej.2016.01.024

Horn SJ, Vaaje-Kolstad G, Westereng B, Eijsink VG (2012) Novel enzymes for the degradation of cellulose. Biotechnol Biofuels 5:45. https://doi.org/10.1186/1754-6834-5-45

Isaksen T, Westereng B, Aachmann FL et al (2014) A C4-oxidizing lytic polysaccharide monooxygenase cleaving both cellulose and cello-oligosaccharides. J Biol Chem 289:2632-2642. https://doi.org/10.1074/jbc.M113.530196

Kittl R, Kracher D, Burgstaller D et al (2012) Production of four Neurospora crassa lytic polysaccharide monooxygenases in Pichia pastoris monitored by a fluorimetric assay. Biotechnol Biofuels 5:79. https://doi.org/10.1186/17546834-5-79

Kracher D, Andlar M, Furtmüller PG, Ludwig R (2017) Activesite copper reduction promotes substrate binding of fungal lytic polysaccharide monooxygenase and reduces stability. J Biol Chem 293:1676. https://doi.org/10.1074/jbc.RA117. 000109

Kračun SK, Schückel J, Westereng B et al (2015) A new generation of versatile chromogenic substrates for highthroughput analysis of biomass-degrading enzymes. Biotechnol Biofuels 8:70. https://doi.org/10.1186/s13068015-0250-y

Quinlan RJ, Sweeney MD, Lo Leggio L et al (2011) Insights into the oxidative degradation of cellulose by a copper metalloenzyme that exploits biomass components. Proc Natl Acad Sci 108:15079-15084. https://doi.org/10.1073/pnas. 1105776108

Singh RK, Blossom BM, Russo DA et al (2019) Thermal unfolding and refolding of a lytic polysaccharide monooxygenase from Thermoascus aurantiacus. RSC Adv 9:29734-29742. https://doi.org/10.1039/C9RA05920B

Vaaje-Kolstad G, Westereng B, Horn SJ et al (2010) An oxidative enzyme boosting the enzymatic conversion of recalcitrant polysaccharides. Science 330:219-222. https:// doi.org/10.1126/science.1192231

Vuong TV, Liu B, Sandgren M, Master ER (2017) Microplatebased detection of lytic polysaccharide monooxygenase activity by fluorescence-labeling of insoluble oxidized products. Biomacromol 18:610-616. https://doi.org/10. 1021/acs.biomac.6b01790

Walton PH, Davies GJ (2016) On the catalytic mechanisms of lytic polysaccharide monooxygenases. Curr Opin Chem Biol 31:195-207. https://doi.org/10.1016/j.cbpa.2016.04. 001

Wang D, Li J, Wong ACY et al (2018) A colorimetric assay to rapidly determine the activities of lytic polysaccharide monooxygenases. Biotechnol Biofuels 11:215. https://doi. org/10.1186/s13068-018-1211-z

Westereng B, Agger JW, Horn SJ et al (2013) Efficient separation of oxidized cello-oligosaccharides generated by cellulose degrading lytic polysaccharide monooxygenases. 
J Chromatogr A 1271:144-152. https://doi.org/10.1016/J. CHROMA.2012.11.048

Westereng B, Cannella D, Agger JW et al (2015) Enzymatic cellulose oxidation is linked to lignin by long-range electron transfer. Nat Publ Gr. https://doi.org/10.1038/ srep 18561
Publisher's Note Springer Nature remains neutral with regard to jurisdictional claims in published maps and institutional affiliations. 